und Alkohol, sowohl wasserhaltigem als wasserfreiem, in sehr reichlicher Menge löslich, wenig löslich in Aether.

Wird das trockene Kalisalz mit verdïnnter Salzsäure über. gossen, so scheiflet sich das Hydrat des Sulfocarbonats als ölige farblose oder blassgelbliche Flüssigkeit von sehr unangenehmem, durchdringendem Geruche ab, die man iber Chlorcalcium trocknen muss, um sie gegen Zersetzung zu schützen.

Sie röthet stark Lakmus, brennt entzündet mit leuchtender Flamme und ist wenig schwerer als Wasser, da sie zwar in reinem Wasser untersinkt, aber auf der wässrigen Salzauflösung, aus welcher sie abgeschieden wird, schwimmt. Sie färbt die Haut tief gelb.

Mit schwefelsaurem Kupferoxyd liefert die Lösung des Kalisalzes einen citronengelben flockigen Niederschlag; mit essigsaurem Bleioxyd ist der Niederschlag weiss, wird aber beim Kochen schwarz; Silberoxydlösung giebt ebenfalls einen weissen, sowohl beim Stehen am Licht als beim Erwärmen sehr schnell schwarz werdenden Niederschlag. Mit Quecksiberchlorid erhält man einen weissen Niederschlag, der sich beim Erhizen nicht schwärzt.

Man sieht, dass die Eigenschaften des Amylsulfocarbonats auf eine merkwürdige Weise denen der entsprechenden Aethylverbindung älnnlich sind.

\title{
II.
}

\section{Ueber die Bildung des Wachses der Bienen.}

Von

\section{Dumas und Irilne Rawarals.}

(Compt. rend. XVII. No. 12. 18. Sept. 1843.)

Die Bereitung des Wachses durch die Bienen hat schon seit langer Zeit die Aufmerksamkeit der Entomologen gefesselt und ist der Gegenstand zahlreicher Untersuchungen gewesen. Da man in den Pflanzen, welche die Bienen täglich besuchen, häufig eine wachsartige Substand antrift, so hat diess die Beobachter zu 
der Mleinung bewogen, dass die Natur diesen arbeitsamen Insecten nicht die Sorge aufgebürdet habe, die zur Bildung ihrer Waben geeigneten Haterien selbst zu erzeugen, sondern sie dieselben nur angewiesen habe, diese Materien zu sammeln und dann zu verarbeiten. Diess ist nämlich die Ansicht, bei welcher $\mathrm{S}$ w a mmerdamm*), Maraldi $* *$ ) und Réaumur ${ }^{* * *}$ ) sich beruhigt haben. Sie glaubten, dass der Pollen der Blumen, von der Biene gesammelt, so zu sagen das rohe Wachs sei, und dass, um dasselbe fertig zu machen, es nur von einer ans eigenthümlichen Organen der Biene abgesonderten Flüssigkeit durchdrungen würde. Aber die Versuche von Hunter haben uns gelehrt, dass bei der Production des Wachses das Insect keine so einfache Rolle spiele; denn dieser grosse Anatom hat gezeigt, dass diese Mlaterie von den Wünden einer gewissen Anzahl von mit Drüsen versehenen Taschen, welche sich an dem Unterleibe des Insectes belinden, abgesondert werle, und dass dieselbe sich dort in der Form yon Lamellen ansammele $f$ ). Dieses erste Resultat wurde anch von Huber $t+$ ) bestätigt, aber es genügte diesem geistreichen und gewandten Beobachter nicht. Angetrieben durch den Forschungsgeist, von dem $H$ uber den Entomologen ein so schönes Beispiel geliefert hat, versuchte er, ob das auf diese Art von den Bienen abgesonderte Wachs in ihren Nahrungsmitteln schon präexistire und nur durch ihren Körper hindurch gehe, um sich in den Wachsbenteln ihres Unterleibes anzusammeln, oder auch, ob es von diesen Insecten erzeugt und auf Kosten der zuckerhaltigen Substanzen gebildet sei, welche sie aus der Krone der Blumen schöpfen. Um diese Frage zu lösen, schloss er Bìenen in einen Korb ohne Ausgang ein und gab ihnen als Nahrungsmittel nur Honig oder Zucker; die gefangenen Arbeiter fuhren dessenungeachtet fort, ihre Wachswaben zu construiren, und er schloss daraus, dass die Bienen die Fähigkeit hätten, den Zucker in Wachs umzuwandeln.

\footnotetext{
\#) Biblia naturae et Collect. Academ., V. p. 237.

* Observations sur les Abeilles (Mém. de l'Acad. des Scienc. annce 1712.)

***) Mémoire pour servir à l'Histoire des Insectes, V. p. 403.

†) Philosophical Transactions, 1792.

H) Nouvelles observations sur les Abeilles; II. chap. 1.
} 
Dieses Resultat, welches für die Entomologie von so grosser Bedeutsamkeit ist, interessirt den allgemeinen Physiologen nicht weniger lebhaft, da es auf's Engste in Verbindung steht mit einer der wichtigsten Fragen dieser Wissenschaft, mit der Theorie der Ernährung der Thiere. Auch hat man davon Gebrauch gemacht bei einer neuern Discussion, welche vielleicht noch der Academie im Gedächtnisse sein wird, und die Beobachtungen von Grundlach *), der neulich die Versuche von $\mathrm{H}$ uber wiederholt, aber vergessen hat, den Namen seines achtungswerthen Führers anzugeben, sind von Liebig als eins der stärksten Argumente citirt worden zu Gunsten der Ansicht, welche er vertheidigt. Aber die Schlussfolgerungen, welche der berühmte Entomolog ron Genf und sein Nacheiferer von Cassel aus ihren Versuchen gezogen haben, sind vor der Kiritik nicht gesichert gewesen; die Mehrzahl der Chemiker hat dieselben nur mit Bedenken angenommen $*$ ), und es wurde besonders schwer, ein völliges Vertrauen denselben zuzuwenden, seitdem man gesehen hatte, dass alle die durch die Praxis als am günstigsten für die Mïstung der Thiere bewährten Nahrungsmittel solche Quantitäten von fetter Substanz enthalten, als hinlänglich ist, um sich ihre Wirkung zu erklüren, ohne dem Thiere, welches sich durch dieselben ernährt, die Fähigkeit der Erzeugung des Fettes beilegen zu müssen. In der That, um die Schlussfolgerungen von $\mathrm{Hu}$ ber als richtig anerkennen zu lassen, hätte er die in den Körpern der dieser Zuckernahrung ausgesetzten Bienen präexistirende Quantität fetter Substanz nachweisen, dieselbe mit derjenigen des producirten Wachses vergleichen und dann untersuchen miissen, ob die Thiere während des Verlaufes des Versuches nicht abgemagert seien; denn es ist bekannt, dass die Absonderungen im Allgemeinen noch eine gewisse Zeit hindurch fortdauern, selbst dann, wenn die ganze Ernährung unterbrochen worden ist, und dass in diesem Falle dieselben auf Kosten der in den Organen präexistirenden Substanzen bewerkstelligt werden; das in den verschiedenen Theilen des Körpers niedergelegte Fett wird alsdann absorbirt und scheint verwandt za werden, als ob es ein Nahrungsmittel

*) Die Naturgeschichte der Bienen. Cassel $18 \pm 2$.

**) S. B erzelius, Lehrb. d. Chemie, Th. V. - Thénard, Traité de Chimie, IV. p. 477 . 
gewöhnlicher Art wäre. Da nun weder Huber noch Grundlach diese Umstände beachtet haben und man folglich sich fragen muss, ob in den Versuchen dieser beilen Entomologen das abgesonderte Wachs wirklich erst auf Kosten des Zuckers, wovon ihre Bienen sich nährten, erzeugt sei, oder auch, ob dieses nicht schon vorher aus den Planzen gesammelt und im Innern der Körper der Insecten aufbewahrt worden sei, eben so wie dieses mit dem Fette der Fall zu sein scheint, welches in so grosser Menge um die Eingeweide der Mehrzahl der Larven sich ansammelt und welches dann in der Periode der Enthaltsamkeit verschwindet, während welcher die Metamorphose völlig beendet wird.

In der Hoffaung, diese Schwierigkeiten zu heben, liaben wir uns vereinigt, um den beruilmten Versuch von $H$ uber zu wielerholen, indem wir denselben so viel wie möglich mit Hülfe der chemischen Analyse vervollständigten, und indem wir uns so vor den Ursachen zum Irrthum, welche wir angeführt haben, bewalriten.

Nach verschiedenen fruchtlosen Versuchen, welche hier mitzutheilen unnütz wäre, sind wir dahin gelangt, unsere gefangen gehaltenen und einer bestimmten Lebensordnung unterworlenen Bienen zum Arbeiten zu bringen.

Unser erster Yersuch war der Ansicht von $\mathrm{Hub}$ er nicht günstig. Ein Bienenschwarm wurde in einen neuen Korb gebracht und dieser in eine Kammer gestellt, deren Fenster mit einem Gewebe von Metalldraht bekleidet war, und die Bienen wurden durch eine beliebige Menge Zuckercassonade ernährt. Nach einigen Tagen der Gefangenschaft fingen die Arbeitsbienen an zu arbeiten und machten zwei kleine Wachswaben; aber ihre Thätigkeit war nur von kurzer Dauer, und es schien bald, dass es ihnen unmöglich sei, in der Production von Wachs fortzufahren, obgleich sie erst eine sehr geringe Quantitït desselben geliefert hatten. Die beiden Kuchen nümlich wogen nur 4,284 Gr. und gaben nur 3,5 Gr. reines Wachs; die Bienen, welche zu der Productinn desselben beigetragen hatten, waren der Zahl nach 5615 zugegen. Jede Arbeitsbiene hatte also im Mittel ungefähr ein halbes Milligramm Wachs geliefert, und die Analyse einer gewissen Anzahl dieser Insecten, welche vor dem Anfange des 
Versuches war angestellt worden, hatte uns überzeugt, dass der Körper eines jeden derselben ungefähr 2 Milligr. schon gebildeter fetter Substanzen enthalten musste.

Da also unsere Bienen, welche mit Zucker gefüttert wurden, nur so beschränkte Quantitäten Wachs gaben, und die Ursachen zu unvermeidlichen Fehlern bei Untersuchungen solcher Art beträchtlicher sind als die Wirkungen, welche wir zu messen gehalit haben, so schien es. uns unnütz zu sein, noch zu untersuchen, ob die erhaltene fette Substanz während der Dauer dieses Versuches wirklich producirt worden sei oder nicht; wir haben es vorgezogen, den Versuch zu wiederholen, indem wir die für die Lebensart der Bienen giinstigsten Bedingungen zu Wege brachten, nämlich dadurch, dass wir sie mit Honig ernïhrten, nachdem wir vorher die in diesem Nahrungsmittel enthaltene Quantität Wachs bestimmt hatten.

Vier Bienenschwärme wurden in mit Glasfenstern versehene Körbe gebracht, welche auf die Art eingerichtet waren, dass der zur Ernährung unserer Bienen bestimmte IIonig und das Wasser leicht in dieselben hineingebracht werden konnte. Drei von dicsen Scliwärmen haben keine Spur von Wachs geliefert, obgleich die Ordnung, der man sie unterwarf, ihnen cine genügende Nallrung zu verschaffen schien. Der 4 . Behälter aber gab uns andere Resultate.

Der Schwarm, welcher Gegenstand dieses Versuches war, halte in seinem alten Korbe mehrere Wachswaben gebildet, aber er war sehr schwach, da er nur 2005 Arbeitsbienen enthielt. Den 7. Juli nahmen wir sie aus dem Korbe heraus, nachdem wir 117 zur Analyse bestimmte Individuen von ihnen getrennt hatten, um uns über die in den Körpern dieser Insecten schon präexistirende Menge fetter Substanz Aulklärung zu verschaffen.

Wir erhielten auf diese Art 0,208 Gr. fetter Substanz.

Jede Biene lieferte uns also im Mittel $0,0018 \mathrm{Gr}$. fetter Substanz *), und wenn man dieses Resultat für die Bestimmung

*) Diese Bestimmung weicht sehr wenig ab von den Resultaten, welche die Analyse einer bestimmten Anzahl Bienen lieferte, die in den 3 anderen Körben fast denselben Bedingungen ausgesetzt wurden. Nïmlich in dem eínen von diesen Versuchen fanden wir im Mittel genommen $0,0017 \mathrm{Gr}$. an fetter Subatanz fir jedes Individuum, in dem zweiten 0,0021 und in dem dritten 0,002 . 
der in dem Kïrper der übrigen 1788 Arbeitsbienen enthaltenen anwendet, so sieht man, dass die Totalmasse an fetter Substanz, welche unser gefangener Schwarm enthielt, nicht über 3,219 Gr. betragen konnte.

Der unsern Bienen zur Nahrung bestimmte Honig enthielt nach unserer Analyse dem Gewichte nach 108000 wachsartiger Substanz. Während der ersten 10 Tage des Versuches brachten wir 411,779 Gr. dieses Nahrungsmittels in unsern Bienenkorb hinein, und folglich gaben wir unsern Arbeitern 0,329 Gr. an fetter Materie, die der zuckerartigen Substanz beigemischt war.

Kurze Zeit nach ihrer Einsperrung fingen die Bienen an zu arbeiten, und den 18. Juli, also am 12. Tage des Versuches, nahmen wir aus dem Korbe 3 Kuchen heraus, deren rohes Gewicht ungefähr 17 Grammen betrug and deren Zellen Eier oder Larven enthielten. An den folgenden Tagen zeigten unsere Bienen eine grosse Unruhe, verïnderten oft ihren Platz und fingen nicht an von Neuem zu arbeiten; aber diese Unterbrechung in ihrer Thïtigkeit schien nicht auf Mangel an Materialien zum Bauen zu beruhen, denn es fiel von dem Unterleibe unserer Arbeiter eine beträchtliche Menge von Wachslamellen ab, welche wir sorgfältig sammelten und zu den vorher erhaltenen Kuchen hinzuthaten. Der Versuch wurde bis zum 8. August fortgesetzt, und während dieser zweiten Periode verzehrten unsere Bienen 423,110 Gr. Honig; viele starben und zuletzt hörten sie auf, sich gruppenweise zu vereinigen, welches sie immer thun, wenn sie die Waben construiren wollen.

Um die wirkliche Quantität an wachsartiger Substanz zu bestimmen, sowohl in den Kuchen selbst, als in dem Körper der Larven und in den auf dem Boden des Korbes zerstreut umherliegenden Lamellen, vertrocknete man zuerst die Larven im Wasserbade, darauf behandelte man das Ganze, zu drei wiederholten Malen, mit kochendem. Wasser, um die zuckerartige Materie und einige andere fremdartige Substanzen aufzulösen. Die so gereinigte Masse wurde der Einwirkung des kalten Alkohols unterworfen, welcher bei der Verdampfung im Wasserbade einen Rückstand hinterliess, der $0,064 \mathrm{Gr}$. wog und identisch zu sein schien mit der vorher aus den Bienen ausgezogenen fetten Materie. Endlich wurde der bei der Einwirkung des Alkohols bleibende 
Rückstand mit kochendem Aether behandelt, und diese Flüssigkeit, zuerst im Wasserbade und darauf im 0elbade bei 140 Centigraden abgedampft, hinterliess $11,451 \mathrm{Gr}$. reines Wachs.

Das ganze Gewicht des von unsern Bienen gelieferten Wachses betrug also 11,515 Gr., welches, getheilt durch die Anzahl der Arbeitsbienen, im Mittel für die Production eines jeden Individuums $0,0064 \mathrm{Gr}$. ausmacht. Diese Quantität ist also, wie man sieht, weit grösser als die in dem Organismus unserer Insecten beim Anfange des Versuches präexistirende Menge an fetter Materie, oder als die in ihren Körper mit dem Honig, womit wir sie ernährt haben, hineingebrachte. Aber um die Resultate noch genügender zu machen, musste.. wir noch die Menge an Fett bestimmen, welche noch in dem Innern unserer Arbeiter übrig sein konnte, nachdem sie das Wachs, dessen wir Erwïhnung gethan haben, geliefert hatten. Einige Tage nachdem die Arbeiten aufgehört hatten, nahmen wir daher aus dem Korbe 105 geschlechtslose Bienen heraus, um dieselben zu analysiren. Weit entfernt, von der Lebensart, der wir sie unterworfen hatten, gelitten zu haben, befanden sie sich im Gegentheil in sehr gutem Zustande und schienen fetter geworden zu sein, denn sie wogen 13,418 Gr.; diess macht für jedes Individuum 0,1277, während vor dem Versuche das Gewicht einer jeden Biene im Mittel nur $0,087 \mathrm{Gr}$. betrug. Man konnte leicht das in den Taschen des Unterleibes angesammelte Wachs wahrnehmen, und die 8 Lamellen, welche wir von einem Individuum abnahmen, wogen 0,0015 Gr. Die Körper dieser Bienen wurden, nachdem sie im Wasserbade eingetrocknet worden waren, auf dieselbe Art behandelt wie bei der ersten Analyse, und man erhielt 0,442 Gr. an fetter Materie, wovon also auf jedes Individuum 0,0042 Gr. kommen.

Bei der Beendigung des Versuches, am 8. August, machten wir von Neuem eine Analyse mit unsern Bienen. Es wurde mit 504 Individuen operirt, deren mittleres Gewicht für jedes Individuum 0,106 Gr. betrug, und man fand, dass ein jedes noch im Mittel 0,004 Gr. fetter Substanz enthielt; diese Insecten waren also während der zweiten Periode ihrer Gefangenschaft ein wenig abgemagert, aber diess erklärt sich leicht aus der grossen Unruhe, in welcher sie sich damals befanden. Indess enthielten sie noch 
mehr als doppelt so viel an fetter Substanz, als am Anfange des Versuches.

Wenn man die so eben angeführten Zahlen betrachtet, so sieht man, dass die Quantitiit der in dem Organismus am Anfange des Versuches präexistirenden fettartigen Substanzen durchaus ungenügend ist, um die Production des Wachses, welche wir nachgewiesen haben, zu erklären. Nümlich:

Die in dem Körper einer jeden Biene prïexistirende fette Materie ist bestimmt worden zu . $0,0018 \mathrm{Gr}$.

Die einer jeden Arbeitsbiene während der ganzen Dauer des Versuches gelieferte fette Substanz betrug . 0,00038

Also die Totalmenge an fetter Materie, deren Ursprung der Nahrung zugeschrieben werden könnte, betrïgt noch nicht für jede Biene

Nun hat wiihrend des Verlaufes des Versuches jeder Arbeiter eine Quantitiat Wachs producirt von . . . . . . . . . . . . 0,0064.

Und nach dieser beträchtlichen Absonderung enthielt jede Biene noch im Innern ihres Körpers, sowohl an Wachs wie an gewöhnlichem Fette

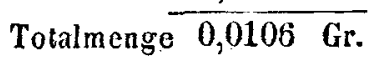

Sobald die Jahreszeit es uns erlauben wird, haben wir uns vorgenommen, diesen Versuch in einem grössern Massstabe zu wiederholen; aber die Thatsachen, welche wir mitgetheilt haben, scheinen uns deutlich zu zeigen, dass die Bienen, unter dem Einflusse einer aus reinem Honig bestehenden Nahrung, wirklich Wachs produciren.

Die Production des Wachses beruht also auf einer wirklichen animalischen Secretion, und die Ansicht über diesen Gegenstand von den älteren Naturforschern, so wie die einiger neuerer Chemiker, unter deren Zahl auch Einer von uns sich rechnen zu müssen geglaubt hat, muss verworfen werden. Die schöne Beobachtung von Huber, uber die Umwandlung des Zuckers in Wachs, findet sich im Gegentheil bestätigt, und wir schätzen uns glücklich, die ersten gewesen zu sein, welche die Zweifel völlig verschwinden lassen, die uns hindern, die Resultate dieses ge- 
wandten Beobachters, so wie die Folgerungen, welche sich daraus ableiten lassen, anzuerkennen. In dem Augenblicke, in welchem die Chemie mehr und mehr in das Gebiet der Physiologie eindringt, müssen alle Meinungen der Untersuchung mittelst der Wage unterworfen werden, welche die Wahrheit von dem Irrthume scheiden und uns erkennen lassen wird, in welchen Fällen ein einfacher Uebergang der Nahrungsstoffe in die Süfte des Körpers stattlindet, und in welchen Fällen diese Substanzen dagegen sich unter dem Einflusse des Organismus modificiren oder ganz umwandeln.

Es ist sehr wahrscheinlich, dass gewisse fette Substanzen von einer besondern Beschaffenheit, wie zum Beispiel das Cholesterin, analogen Einwirknngen ihre Entstehung verdanken, als diejenigen sind, welche die Bildung des Wachses veranlassen; aber diese Frage kann nur aul dem Wege der Erfahrung und des Versuches entschieden werden.

\section{III. \\ Ueber das chinesische Wachs. \\ Von}

B. $\boldsymbol{L} \boldsymbol{e w} \boldsymbol{y}$.

(Compt. rend. XVII. No.18. 30. Oct. 1843,)

Da ich eine allgemeine Untersuchung über die verschiedenen Wachssorten unternommen habe, so hat Herr Dumas die Gefälligkeit gehabt, mir die Untersuchung einer Probe des chinesischen Wachses (Rhus succedaneum) anzuvertrauen, welche er der Güte des Herrn $\mathbf{S}$ tanis las Jul i en verdankt.

Diese Substanz, welche vegetabilischen Ursprungs ist, hat nicht dasselbe Aussehen wie das Wachs der Bienen; sie ist glänzend weiss, krystallinisch, und gleicht, nach ihren äusseren Eigenschaften, dem Wallrath.

Sie schmilat bei $82,5^{\circ} \mathrm{C}$. 